\title{
Rechtsunsicherheit und Rent-Seeking in autoritären Regimen
}

\author{
Von Rupert F.J. Pritzl ${ }^{1}$
}

\section{Einleitung}

Für eine Analyse der gesellschaftlichen Verteilungskonflikte in vielen Ländern der Dritten Welt läßt sich das Konzept des Rent-Seeking sinnvoll anwenden. Unter Rent-Seeking kann man vereinfachend die vielfältigen Bemühungen von Individuen oder Gruppen verstehen, staatliche Eingriffe in die Allokation herbeizuführen und sich die hierdurch künstlich geschaffenen (Renten-) Einnahmen anzueignen. Eine Analyse der vielfältigen RentSeeking-Aktivitäten sowie der hierdurch hervorgerufenen Rechtsunsicherheit kann dazu beitragen zu erklären, warum in vielen Entwicklungsländern in den zurückliegenden Jahrzehnten wirtschaftliches Wachstum und gesellschaftliche Entwicklung vielfach ausgeblieben sind und notwendige Maßnahmen der Struktur- und Anpassungsprogramme nicht oder nur unzureichend durchgeführt wurden. ${ }^{2}$ Hierbei wird es von besonderer Bedeutung sein, auf die Funktionsweise autoritärer politischer Systeme kurz einzugehen, in denen legales, aber auch illegales Rent-Seeking notwendige politische Elemente bilden und selbst ein unfriedliches Rent-Seeking möglich ist. ${ }^{3}$

\section{Sichtweise und Instrumentarium der Neuen Institutionenökonomik}

Hier wird auf grundlegende Elemente der Neuen Institutionenökonomik zurückgegriffen (u.a. Rent-Seeking, Verfügungs- und Nutzungsrechte ('Property Rights') und Theorie der Interessengruppen), die eine interdisziplinäre Analyse der gesellschaftlichen Entwicklungshemmnisse möglich macht. ${ }^{4}$ Die verfügbaren Güter werden als Bündel von Rechten angesehen, die aus den Rechten auf Nutzung (usus), auf Einbehaltung der Erträge (usus fruc-

1

2

3

4
Der Beitrag gibt die persönliche Meinung des Autors wieder.

Vgl. Weltbank, Governance and Development, Washington D.C. 1992, S. 4 ff.

Vgl. Rupert F.J. Pritzl, Korruption und Rent-Seeking in Lateinamerika. Zur Politischen Ökonomie autoritärer politischer Systeme, Baden-Baden 1997.

Vgl. Rudolf Richter / Eirik Furbotn, Neue Institutionenökonomik: Eine Einführung und kritische Würdigung, Tübingen 1996; Rupert F.J. Pritzl, Entwicklungsperspektiven für Lateinamerika eine institutionenökonomische Analyse, in: Politische Studien, Heft 348, 47. Jhg., Juli/August 1996, S. 24-43, und Mustapha K. Nabli / Jeffrey' B. Nugent, The New Institutional Economics and Its Applicability to Development, in: World Development, Vol. 17 (9), 1989, S. 1333-1347. 
tus), auf Veränderung von Form und Substanz (abusus) und auf Übertragung (Transfer) bestehen. Der Tausch von Gütern entspricht dem Tausch von diesen Nutzungs- und Verfügungsrechten. Da der Wert der Güter in erheblichem Maße von der Ausgestaltung dieser Verfügungs- und Nutzungsrechte abhängt, hat ein Eingriff in dieses Rechtsbündel oder eine Änderung des Systems der Nutzungsrechte (z.B. durch staatliche Intervention) einen spezifischen und in der Tendenz vorhersehbaren Einfluß auf die Allokation der Ressourcen und auf die Verteilung des Einkommens. So erhöht sich z.B. der Wert eines Unternehmens beträchtlich, wenn es eine Ausnahmegenehmigung von den allgemeinen Import- oder Exportbeschränkungen oder spezielle Vergünstigungen erhält.

Unter einer 'Institution' werden alle verhaltensleitenden und erwartungsstabilisierenden Strukturen verstanden, die dazu beitragen, das Verhalten der Individuen zu verstetigen und für andere vorhersehbar zu machen. Die Institutionen eröffnen einerseits Handlungsmöglichkeiten für die Individuen, beschränken andererseits aber auch deren Handlungsspielräume. Da die Institutionen reziproke Verhaltenserwartungen der Individuen ermöglichen, wird so die gesellschaftliche Unsicherheit verringert.

Wenn man von den zentralen Verhaltensannahmen der ökonomischen Theorie ausgeht (u.a. zweckrationales und nutzenmaximierendes Verhalten), so wird deutlich, daß sich das Verhalten der Individuen aus ihren individuellen Präferenzen sowie den institutionellen Strukturen als Verhaltensmöglichkeiten und -beschränkungen ergibt. Aus diesem Grund steht bei der Neuen Institutionenökonomik die Analyse des institutionellen gesellschaftlichen Umfeldes im Vordergrund, zu dem auch die Art des politischen Systems zählt.

\section{Das Konzept des Rent-Seeking}

Das Konzept des Rent-Seeking basiert auf wettbewerbs- und regulierungstheoretischen Vorstellungen und erlaubt die Analyse der gesellschaftlichen Kosten, die von diskretionären staatlichen Eingriffen in den Wirtschaftsprozeß hervorgerufen werden (z.B. staatlicher Eingriff zur Schaffung von Monopolen). Als Rente werden die Zahlungen an den Rechtsinhaber einer Ressource definiert, die über den Einkommen liegen, die in der nächstbesten Verwendung der Ressourcen erzielt werden können. ${ }^{5}$ Renten entstehen vor allem dort, wo staatliche Eingriffe und Regulierungen direkt oder indirekt zu künstlichen Wettbewerbsund Preisverzerrungen führen und somit Einkommens- oder Transferzahlungen staatlicherseits garantieren. Wenn Politiker und Bürokraten in den marktlichen Prozeß diskretionär eingreifen, schaffen sie viele neue oder verändern bereits bestehende Rechtspositionen und 
erzeugen so staatlich gewährte Renteneinnahmen (z.B. Vergabe von Lizenzen oder Monopositionen).

Die auf Mancur Olson zurückgehende Theorie des kollektiven Handelns läßt sich heranziehen, um die Dynamik der Umverteilungsbemühungen der vielfältigen gesellschaftlichen

Interessengruppen näher zu analysieren. ${ }^{6}$ Unter einer Interessengruppe läßt sich der formale oder informelle Zusammenschluß von Individuen verstehen, die weitgehend ähnliche Interessen verfolgen. $\mathrm{Zu}$ den wichtigen gesellschaftlichen Interessengruppen, die sich in den gesellschaftlichen Verteilungskonflikten um eine gruppenspezifische Privilegierung bemühen, gehören z.B. die Unternehmerverbände, die - häufig staatlichen oder zumindest staatsnahen - Gewerkschaften, die politischen Parteien oder Gruppierungen, die - in vielen Ländern wichtigen - traditionellen Großgrundbesitzer sowie die Polizei und das Militär. Auch die Politiker und Bürokraten sind relativ homogene gesellschaftliche Gruppen, die in den gesellschaftlichen Verteilungskonflikten auftreten.

\section{Die gesellschaftliche Bedeutung des Rent-Seeking}

Aus institutionenökonomischer Sicht läßt sich das Rent-Seeking als die Bemühungen auffassen, im politisch-bürokratischen Prozeß individuell oder kollektiv eine Veränderung der Verfügungs- und Nutzungsrechte zu seinen Gunsten zu erreichen. Während es auf dem ökonomischen Markt zu einem in der Regel freiwilligen Tausch von Rechten zwischen prinzipiell gleichgeordneten Wirtschaftssubjekten kommt, erfolgt auf dem 'politischen Markt' eine Veränderung der Verfügungs- und Nutzungsrechte durch Akteure, die anderen hoheitlich übergeordnet sind. Beim Rent-Seeking geht es daher um die Suche nach und die Aneignung von politischen (Renten-) Einkommen.

Die hoheitliche Macht zur Veränderung der Eigentumsrechte besitzen die Politiker und Bürokraten, die per Gesetz Dispositionen vornehmen können, die für die übrigen Wirtschaftssubjekte verbindlich sind und - falls erforderlich - auch mit staatlicher Zwangsgewalt durchgesetzt werden können. Auf diese Weise können die hoheitlichen Akteure in erheblichem Umfang einseitig auf die Verfügungs- und Nutzungsrechte der Individuen Einfluß nehmen. Da den Politikern und Bürokraten zum Teil erhebliche Ermessens- und Entscheidungsspielräume eingeräumt werden, innerhalb derer ihre hoheitlichen Handlungen nur unzureichend kontrolliert werden können, verbleiben ihnen relativ große und willkürlich nutzbare Handlungsspielräume. Auf diese Weise werden die Politiker und Büro-

6

Vgl. Mancur Olson, The Logic of Collective Action. Public Goods and the Theory of Groups, Harvard University Press 1965 und derselbe, The Rise and Decline of Nations. Economic Growth, Stagflation and Social Rigidies, New Haven 1982 (dt. Ausgabe: Aufstieg und Niedergang von Nationen. Ökonomisches Wachstum, Stagflation und soziale Starrheit, Tübingen, 2. Aufl. 1991). 
kraten die zentralen Akteure in den gesellschaftlichen Umverteilungsbemühungen, bei denen das Zusammenspiel zwischen den Verfügungs- und Nutzungsrechten und dem RentSeeking von zentraler Bedeutung ist.

Wenn die Interessengruppen die Möglichkeit haben, an die Politiker und Bürokraten heranzutreten und diese zu politischen Eingriffen zu ihren Gunsten zu veranlassen, so ist realistischerweise anzunehmen, daß diese Gruppen die Möglichkeiten auch tatsächlich nutzen werden. Die volkswirtschaftlichen Ressourcen werden dann von den eigentlichen produktiven Tätigkeiten auf dem ökonomischen Markt abgezogen und in den 'politischen Markt' zur legalen - oder auch illegalen - Beeinflussung der politischen Entscheidungsträger gelenkt. ${ }^{7}$ Da das Rent-Seeking letztlich nur eine 'aufwendige' Umverteilung der bisherigen Einkommen darstellt, ist es gesellschaftlich weitgehend unerwünscht, und die Aufwendungen, die hierdurch getätigt weden, sind unproduktiv.

Die Rent-Seeking-Aktivitäten werden dann einen erheblichen Umfang erreichen, wenn die gesellschaftlichen Institutionen (z.B. das politische System), die Art der betriebenen Wirtschaftspolitik und das Handeln der Repräsentanten des Staates dazu beitragen, daß diese 'politischen' Aktivitäten auch ökonomisch belohnt werden. Hierzu tragen die 'Schwächen' der politischen Systeme in vielen Ländern der Dritten Welt (so u.a. die fehlende oder unzureichende Gewaltenteilung, fehlende rechtsstaatliche Elemente sowie die politische Abhängigkeit der Judikative ['Politisierung der Justiz']) in erheblichem Maße bei. Daher sind gerade in den Ländern umfangreiche Rent-Seeking-Aktivitäten zu erwarten, in denen z.B. umfangreiche staatliche Eingriffe in und Regulierungen des marktlichen Wettbewerbs vorgenommen, eine Vielzahl von staatlichen bzw. halbstaatlichen Unternehmungen betrieben werden sowie ein ausgedehnter öf fentlicher Sektor unterhalten wird. Je unkontrollierter und willkürlicher die politischen Entscheidungsträger handeln (können), desto weniger vorhersehbar wird deren Verhalten und desto größer wird die gesellschaftliche Unsicherheit. Die gesellschaftliche Unsicherheit wird dann ein Höchstmaß erreichen, wenn die staatlichen Repräsentanten weder eine rechtsstaatliche (durch eine unabhängige Judikative) noch eine demokratische Kontrolle (über demokratische Wahlen, eine aktive Öffentlichkeit und investigative Presse) zu befürchten haben.

\section{Rent-Seeking bei gesellschaftlicher Unsicherheit}

Für viele Entwicklungsländer ist eine Divergenz zwischen dem geschriebenen ('de jure') und dem tatsächlich geltenden ('de facto') Recht typisch. Diese kommt z.B. darin zum

Die öffentliche Korruption ist als eine solche Form des illegalen Rent-Seeking anzusehen (vgl. John M. Mbaku, Bureaucratic Corruption as Rent-Seeking Behavior, in: Konjunkturpolitik, Vol. 38 (4), 1992, S. 247-265). 
Ausdruck, daß vielfach Unklarheit hinsichtlich der Existenz und der Geltung der Eigentumsrechte vorliegt. In vielen Fällen garantiert ein formaler Eigentumstitel dem Rechtsinhaber noch lange nicht die tatsächliche und eine weitgehend gesicherte Nutzungsbefugnis. Vielfach herrscht auch Unsicherheit hinsichtlich der Rechtsetzung der Legislative, der Rechtsanwendung und der Rechtsauslegung der Exekutive sowie der richterlichen Rechtsprechung vor. ${ }^{8}$

Wenn das Rechtswesen nicht hinreichend für die tatsächliche Durchsetzung der Eigentumsrechte sorgen kann, gewinnen die politischen Entscheidungsträger zunehmende Bedeutung auch für die Durchsetzung dieser Rechte. Man wendet sich folglich an die Politiker und Bürokraten mit dem Wunsch, die Eigentumsrechte auf politischem Wege durchzusetzen. Auf diese Weise werden die Individuen und die gesellschaftlichen Gruppen vor dem Beschreiten des Rechtsweges von vornherein zurückschrecken und sich sowohl für die Zuerkennung als auch für die Durchsetzung von Eigentumsrechten an die politischen Entscheidungsträger wenden. Ein politisiertes Rechtssystem ist dann ein von den Politikern privatisiertes Rechtssystem, in dem die Politiker die (Rechts-)Regeln und auch die Ergebnisse (willkürlich) bestimmen können.

Es ist aber auch möglich, daß bestimmte gesellschaftliche Gruppen sich ein privates Machtund Gewaltpotential aufbauen und ihre Forderungen in den gesellschaftlichen Verteilungskonflikten mit unfriedlichen Mitteln durchsetzen. Das legale Rechtssystem wird dann durch die 'Privat'justiz ersetzt und die Eigentumsrechte mit privater Gewalt durchgesetzt. In den Ländern, in denen der Staat nicht willens oder nicht fähig ist, das gesellschaftliche Machtund Gewaltpotential zu monopolisieren, wird die illegale Gewaltanwendung zu einem zentralen Mittel in den gesellschaftlichen Verteilungskonflikten. Es ist offensichtlich, daß bei einem solchen unfriedlichen Rent-Seeking vor allem die Militärs, die Polizei - aber auch in manchen Fällen in Lateinamerika die traditionellen Großgrundbesitzer - einen komparativen Vorteil haben und durchaus als Stategische Gruppen auftreten können. Politisierung und Privatisierung des Rechtssystems führen zu einem Höchstmaß an gesellschaftlicher Unsicherheit.

Für Lateinamerika vgl. z.B. Deutsche Stiftung für Internationale Entwicklung (o.J.), Rechtsstaatlichkeit, Rechtssicherheit und Justizreformen in Lateinamerika, Bericht über ein Round Table vom 28.11.-1.12.1995, Berlin und Maria Dakolias, The Judicial Sector in Latin America \& the Carribean: A Strategy for Reform, Diskussionspapier der Weltbank, New York 1995.

9 Vgl. Pritzl, 1997, S. 226 ff. Zum Konzept der Strategischen Gruppen vgl. Hans-Dieter Evers / Tilman Schiel, Strategische Gruppen: Vergleichende Studie zu Staat, Bürokratie und Klassenbildung in der Dritten Welt, Berlin 1988. 


\section{Zur Funktionsweise autoritärer politischer Systeme}

Ein autoritäres politisches System läßt sich als ein politisches System definieren, in dem die regulären Prinzipien der Machterlangung und -erhaltung sowie der Ausübung staatlicher Macht weitgehend nicht existieren bzw. nicht wirksam sind. In autoritären Systemen müssen die regierenden Politiker sich stets um ihr persönliches Überleben sowie um eine hinreichende politische Unterstützung durch die mächtigen gesellschaftlichen Gruppen bemühen. Hierfür dient der - auch häufig zu beobachtende - Austausch von unmittelbarer materieller Besserstellung gegen politische Unterstützung, der die prinzipielle autoritäre Herrschafts- und Legitimationsstrategie bildet. Die regierenden Politiker werden zur Stabilisierung des autoritären politischen Systems und zur eigenen Machterhaltung tendenziell eine Politik der gezielten staatlichen Umverteilung (v.a. eine spezielle Interessengruppen-[begünstigungs]politik) und somit eine primär redistributiv ausgerichtete staatliche Wirtschaftspolitik betreiben. Die Aufrechterhaltung der politischen Macht wird durch die Vergabe von spezifischen Vorteilen gleichsam 'erkauft'. ${ }^{10}$

Hieraus folgt zwangsläufig, daß sich die Politiker und Bürokraten gegen jegliche Verringerung ihres Zuständigkeitsbereichs und gegen jegliche Begrenzung ihrer willkürlichen Handlungsmöglichkeiten zur Wehr setzen (werden), um sich jederzeit eine machtpolitisch motivierte Veränderung der Eigentumsrechte offenzuhalten. Autoritäre politische Systeme sind daher mit einer gewaltenteiligen Kontrolle und mit rechtsstaatlichen Prinzipien, die die staatliche Gewalt durch die 'rule of law' vorhersehbar machen, grundsätzlich unvereinbar.

Für den wirtschaftlichen Bereich folgt hieraus zwangsläufig, daß die Politiker und Bürokraten keinen unpersönlichen Marktmechanismus und somit eine marktliche Allokation zulassen, sondern sich stets bemühen, möglichst viele gesellschaftliche Entscheidungen zu personalisieren und zu politisieren und so von sich persönlich abhängig zu machen. Auf diese Weise ist es ihnen möglich - und machtpolitisch auch erforderlich -, ihre 'persönliche Allzuständigkeit' in allen wichtigen gesellschaftlichen Fragen aufrechtzuerhalten und ihr Handeln für andere möglichst wenig vorhersehbar zu machen. Die Vielzahl der diskretionären staatlichen Eingriffe in den Wirtschaftsprozeß und die 'Politisierung der Wirtschaft' (u.a. staatliche Devisenbewirtschaftung, umfangreiche Import- und Exportbeschränkungen, staatliche Preisreglementierungen) in vielen Entwicklungsländern sind daher die kaum vermeidbaren Folgen einer solchen autoritären Herrschaftsstrategie. Hierbei werden zwangsläufig auch die politischen Einkommenserzielungsmöglichkeiten gegenüber den ökonomischen erhöht. Und auch die öffentliche Korruption, die sich als ein illegales RentSeeking auffassen läßt, wird so zu einem zentralen Politikelement in diesen Regimen.

10

Vgl. Pritzl, 1997, S. 163 ff. und Rupert Pritzl / Friedrich Schneider, La economía política en los sistemas políticos autocráticos: ?cómo gobierna el homo oeconomicus autocraticus?, in: Contribuciones, 14. Jahrg., Heft 4/ 1997, S. 127-164. 
Während in einer statischen Betrachtung das Rent-Seeking Ausdruck der gesellschaftlichen Verteilungskonflikte um bereits bestehende Rentenpositionen ist, läßt es sich in einer dynamischen Betrachtung als Ausdruck der Bemühungen auffassen, diese rentenversprechenden Positionen durch staatliche Eingriffe herbeizuführen ('Rentenschaffung') bzw. seine einmal erreichte Position gegenüber anderen abzusichern ('Rentenverteidigung'). Die gesellschaftliche Dynamik dieses - wie Hernando De Soto sich treffend ausdrückt - gesellschaftlichen 'Umverteilungskrieges' ${ }^{11}$ kommt darin zum Ausdruck, daß ein einzelner staatlicher Eingriff und die mit ihm verbundene bevorzugte Behandlung (z.B. in Form von direkter Rentengewährung oder Wettbewerbsvorteile) vielfältige weitere Rent-SeekingAktivitäten auf unterschiedlichen Ebenen und zu verschiedenen Zeiten hervorrufen.

Die vielen gesellschaftlichen Gruppen werden sich daher organisieren und an die Politiker und Bürokraten mit distributiven Forderungen herantreten. Diese wiederum werden jenen Interessengruppen in ihren Wünschen nach Privilegierung entgegenkommen, auf die sie machtpolitisch angewiesen sind. Es entsteht ein dynamisches Zusammenspiel von Anbietern von und Nachfragern nach staatlich regulierenden und gleichzeitig rentenverschaffenden Eingriffen, das zwar dazu beiträgt, das autoritäre Regime zu stabilisieren und den Regierenden ihren Machterhalt zu sichern, das aber gleichzeitig den Umfang und die Dichte der staatlichen Regulierung und die Komplexität der gesetzlichen Beschränkungen stark anwachsen läßt. ${ }^{12}$

Die zahlreichen Widerstände gegen grundlegende Reformmaßnahmen zur Deregulierung der Wirtschaft und zur Privatisierung der defizitären Staatsunternehmen lassen sich vielfach auf diese Rentenverteidigungsbemühungen einzelner Interessengruppen zurückführen. Der größte Widerstand kam vielfach nicht von den Ärmsten, sondern vielmehr von denjenigen Bevölkerungsgruppen, die bisher bereits privilegiert waren und die ihre Privilegien durch die Reformmaßnahmen gefährdet sahen. Auf diese Weise wurden in vielen Ländern der Dritten Welt dringend erforderliche Reformmaßnahmen erheblich verzögert bzw. in vielen Fällen ganz verhindert.

\section{Auswirkungen des gesellschaftlichen Rent-Seeking}

Es ist - nicht zuletzt seit Douglass C. North - bekannt, daß die gesellschaftlichen Institutionen das wirtschaftliche Wachstum und die gesellschaftliche Entwicklung maßgeblich

Vgl. Hernando de Soto, El Otro Sendero. La Revolución Informal, Buenos Aires 1987 (zit. nach der dt. Ausgabe: Marktwirtschaft von unten. Die unsichtbare Revolution in Entwicklungsländern, Zürich u.a. 1992, S. 231).

Vgl. Kevin Murphy / Andrei Shleifer / Robert W. Vishny, Why is Rent-Seeking so costly to growth?, in: The American Economic Review, Vol. 83 (2), 1993, S. 409-414. 
(mit-) bestimmen. ${ }^{13}$ Die institutionellen Anreize entscheiden darüber, ob sich die Handlungen der Individuen für die Gesellschaft als prodıktiv (d.h. unternehmerische Tätigkeit im Schumpeterschen Sinne), unproduktiv (d.h. Rent-Seeking-Aktivitäten) oder gar als destruktiv (in der Regel Kriegshandlungen) erweisen. ${ }^{14}$

Wenn die gesellschaftlichen Anreize zu einem unproduktiven Rent-Seeking-Verhalten überwiegen, so wird sich eine 'Rent-Seeking Society, ${ }^{15}$ herausbilden, in der sich langfristig nur noch die unproduktiven Aktivitäten auf dem 'politischen Markt' auszahlen und die volkswirtschaftlichen Ressourcen in diese Verwendung fließen. Es kommt zu einer Verdrängung der wirtschaftlichen Aktivitäten in den informellen Sektor, zu einer umfangreichen Kapitalflucht sowie zu einer langanhaltenden Inflation. Hieraus folgt aber auch, daß die politischen Strukturen verkrusten und die Gesellschaft unfähig wird, auf politische und wirtschaftliche Veränderungen angemessen zu reagieren. ${ }^{16}$ Die späte Reaktion vieler Länder Afrikas, die vielfach erst zu Anfang der 90er Jahre mit Reformen begonnen haben, zeugt von einer solchen gesellschaftlichen Verkrustung.

\section{Politischer Handlungsbedarf}

Die institutionenökonomische Analyse legt eine Verbesserung der institutionellen gesellschaftlichen Anreizstrukturen nahe, um die produktiven ökonomischen Aktivitäten auch gesellschaftlich wieder 'lohnend' zu machen. Dieser zentrale Aspekt wurde angesichts der vielbeachteten makroökonomischen Stabilisierungsmaßnahmen in vielen Ländern der Dritten Welt bisher vielfach nicht hinreichend berücksichtigt. Und auch im 'Washington Consensus' kommen die institutionellen Reformen zu kurz.

Im politischen Bereich sind vor allem die Einführung rechtsstaatlicher und demokratischer Elemente erforderlich. Zentrale Elemente des Rechtsstaates sind die Bindung der staatlichen Gewalt an Recht und Gesetz und eine gewaltenteilige Kontrolle allen staatlichen Handelns, ein funktionsfähiges und unabhängiges Rechtssystem, ein gesetzmäßiges und nachvollziehbares Verwaltungshandeln sowie ein wirksamer Schutz des einzelnen vor der (Über-) Macht des Staates. Die rechtsstaatlichen Elemente sollen dazu beitragen, die

Vgl. Douglass C. North, Structure and Change in Economic History, New York 1981 und derselbe, Institutions, Institutional Change and Economic Performance, Cambridge 1990.

William J. Baumol, Entrepreneurship: Productive, Unproductive, and Destructive, in: Journal of Political Economy, Vol. 98 (5), 1990, S. 893-921.

J.M. Buchanan / R.D. Tollison / G. Tullock (Hrsg.), Toward a Theory of the Rent-Seeking

16 Society, College Station/ Texas 1980.

Vgl. Anne O. Krueger, Virtuous and Vicious Circles in Economic Development, in: American Economic Review, Vol. 83 (2), 1993, S. 351-355. 
Divergenz zwischen dem geschriebenen und dem tatsächlichen Recht zu verringern und das Verhalten der Politiker und Bürokraten vorhersehbarer und berechenbarer zu machen. Die Einführung demokratischer Elemente wird auch dazu führen, daß die Bevölkerung am politischen Prozeß partizipiert und die politisch Handelnden durch (Ab-) Wahl kontrolliert. Auf diese Weise dürfte es langfristig möglich sein, die autoritären Herrschaftsformen zurückzudrängen und die gesellschaftliche Unsicherheit zu verringern. Im Bereich der Entwicklungszusammenarbeit wären hier z.B. Maßnahmen zur rechtspolitischen Beratung und zur Verwaltunghilfe sowie ein verstärkter Politikdialog zu empfehlen. ${ }^{17}$

Die vielfältigen Rent-Seeking-Aktivitäten und der Einfluß der Interessengruppen können dauerhaft nur dadurch verringert werden, daß sich der Staat selbst in seinem Einflußbereich und in seiner Machtfülle glaubhaft begrenzt und jegliche diskretionären Eingriffe unterläßt, die bestimmte gesellschaftliche Gruppen besserstellen können. Im wirtschaftlichen Bereich wären marktliche und dezentrale Strukturen einzuführen, das Wirtschaftsleben weitgehend zu liberalisieren und zu deregulieren sowie die - meist defizitären - Staatsbetriebe zu privatisieren. Nur auf diese Weise ist es möglich, die unproduktiven politischen (Renten-) Einkommen zu verringern und die produktiven wirtschaftlichen Eigenanstrengungen zu belohnen. Ziel aller wirtschaftspolitischen Reformmaßnahmen muß es sein, die Wirtschaft zu entpolitisieren und die politischen Entscheidungen zu entpersonalisieren. Dieses wird nur dann möglich sein, wenn sich der Staat auf seine eigentlichen Aufgaben konzentriert und sich nicht zum 'Spielball' der organisierten Interessengruppen macht. Letztlich dürfte es in entscheidendem Maße darauf ankommen, daß eine Gesellschaft einen gesellschaftlichen Lernprozeß in Gang setzt und die für sie entwicklungsadäquaten gesellschaftlichen Institutionen selbst entwickelt. Dieser gesellschaftliche Lernprozeß kann von außen zwar unterstützt werden, ohne eine tatsächliche interne Reformbereitschaft dürfte er aber kaum möglich sein. Lateinamerika tätig ist, bereits auf erste ermutigende Erfolge verweisen (vgl. Matthias Herdegen, Deutschland ein wichtiges Vorbild/ In Lateinamerika kommt der Aufbau rechtsstaatlicher Verhältnisse trotz aller Widrigkeiten voran, in: F.A.Z., Nr. 52, vom 3. März 1997, S. 13). 


\title{
ABSTRACTS
}

\section{Corruption and Control of Corruption in Development Co-operation}

\author{
By Georg Cremer
}

Development aid programs are implemented under conditions which bear a high risk that one or the other form of corruption, especially embezzlement, may occur. In many developing countries, corruption is an everyday phenomenon. Development aid organizations do not speak about this problem in public and researchers have only limited empirical access to this topic. This article focuses on embezzlement as an internal problem of development aid, i.e. funds are embezzled by the organizations implementing the programs or by their employees.

Embezzlement of development aid occurs in the governmental sector but also in development programs of non-governmental organizations. Methods of control usually applied in development aid are based on budgetary regulations, but are insufficient to control corruption and must be supplemented by other methods. This paper discusses structural components of the work of development aid donors which make it difficult for them to cope with the problem of corruption in development aid: Disbursement pressure and information barriers inside the organizations are two of these components. Donors encourage the development of structures within their non-governmental partners similar to those in governmental settings if they ignore the legitimate institutional interests of their partners.

\section{Juridical Insecurity and Rent-Seeking in Authoritarian Regimes}

\section{By Rupert Pritzl}

The concept of rent-seeking can be applied to analyze the frequent distributional struggles in many Third World countriess and to explain the resistance to basic structural reforms and the of ten insufficient success in developing these countries.

Based on the New Institutional Economics, the article analyzes the importance of rentseeking in authoritarian regimes. "Rent-seeking" describes the efforts of special interest groups to obtain money worth privileges by exercising their influence on politicians and bureaucrats in the political process. Authoritarian regimes can be defined as political systems in which regular principles of acquiring, maintaining and execution power are not existent or not obeyed. Authoritarian rulers try to ensure their personal and political sur- 
vival by covering themselves of the support of important interest groups and at the same time of deterring others from taking over power.

Personalization of political decisions and politicization of economy and administration as well as personalization and privatization of the juridical sector are necessary political elements to maintain power in authoritarian regimes leading all to and requiring widespread juridical insecurity. As economic and political development can hardly be expected in such authoritarian regimes, the introduction of more democratic and constitution based elements and the implementation of market economic structures are necessary.

\title{
Constitutional Jurisdiction in Guatemala
}

\author{
By Francisco Fernández Segado
}

In Guatemala, the peace treaty signed on December 29, 1996 by President Alvaro Arzú, heading the peace-commission COPAZ, and by the leaders of the URNG, merging four major guerilla-groups, ended a six-year peace marathon which followed 36 years of armed conflict, the last contemporary armed conflict to be solved in Central America.

Nevertheless, 12 years ago, on July 1, 1984, general, fair and free elections for a constitutional assembly took place. This constituante managed to draw up a new constitution within 10 months, which was then promulgated on May 31, 1985. The paper focuses on the newly created constitutional court of Guatemala and, in comparison with the preceding constitution of 1966, on the legal framework attributing this court the powers to protect both human rights and the constitutionality of the political process in general.

The author gives an extensive overview which may serve as a solid base for the upcoming discussion, whether or not democratically legitimated constitutional jurisprudence is able to play an important role in enforcing the peace treaty implementation process within the country.

\section{Popular Action in defense of "Divine Law": Hisba in contemporary Egypt}

\section{By Kilian Bälz}

The paper investigates the Islamic principle of hisba in contemporary Egypt. In examining recent Egyptian case-law, the paper argues that the traditional concept of hisba, the Islamic duty to "promote good and prevent evil", underwent a fundamental transformation: traditionally, the duty of hisba rested upon the public function of the muhtasib ["market supervisor"]. Today, in contrast, the principle is employed to entitle a third party to an action in 